\title{
Evaluation of a New, Rapid, Fully Automated Assay for the Measurement of ADAMTS13 Activity
}

\author{
Carla Valsecchi ${ }^{1}$ Maribel Mirabet ${ }^{2} \quad$ Ilaria Mancini ${ }^{3 \oplus}$ Marina Biganzoli ${ }^{1} \quad$ Lucia Schiavone $^{1}$ \\ Susanna Faraudo ${ }^{4}$ Daniel Mane-Padros ${ }^{4}$ David Giles ${ }^{2}$ Josep Serra-Domenech ${ }^{2}$ Silvia Blanch ${ }^{2}$ \\ Silvia Maria Trisolini ${ }^{5}$ Luca Facchini ${ }^{6}$ Erminia Rinaldi ${ }^{7}$ Flora Peyvandi ${ }^{1,3}$ (1)
} ${ }^{1}$ Fondazione IRCCS Ca' Granda Ospedale Maggiore Policlinico Milano,
A. Bianchi Bonomi Hemophilia and Thrombosis Center and
Fondazione Luigi Villa, Milan, Italy
2 Assay Development Department, Biokit, Barcelona, Spain
${ }^{3}$ Department of Pathophysiology and Transplantation, Università
degli Studi di Milano, Milan, Italy
${ }^{4}$ Clinical Affairs Department, Biokit, Barcelona, Spain
${ }^{5}$ Division of Hematology, Department of Cellular Biotechnologies and
Hematology, Policlinico Umberto 1, Sapienza University, Rome, Italy
6 Hematology Unit, Oncology Department, Azienda Unità Sanitaria
Locale-IRCCS di Reggio Emilia, Reggio Emilia, Italy
${ }^{7}$ Hematology Unit, A. Perrino Hospital, Brindisi, Italy

Thromb Haemost 2019;119:1767-1772.

\begin{abstract}
Address for correspondence Flora Peyvandi, MD, PhD, Fondazione IRCCS Ca' Granda Ospedale Maggiore Policlinico Milano, A. Bianchi Bonomi Hemophilia and Thrombosis Center and Fondazione Luigi Villa, Department of Pathophysiology and Transplantation, Università degli Studi di Milano, Via Pace 9, 20122 Milan, Italy (e-mail: flora.peyvandi@unimi.it).
\end{abstract}

\section{Abstract}

Keywords

- ADAMTS/ADAMTS13

- thrombotic thrombocytopenic purpura (TTP/HUS)

- diagnosis management

- autoimmune diseases

- chemiluminescent assay
Thrombotic thrombocytopenic purpura (TTP) is a rare thrombotic microangiopathy (TMA) characterized by the severe deficiency of a disintegrin and metalloproteinase with a thrombospondin type 1 motif, member 13 (ADAMTS13) activity (<10\%). Rapid ADAMTS13 testing is crucial for an early diagnosis and optimal management of acute TTP. We evaluated the performance of the HemosIL AcuStar ADAMTS13 activity assay (Instrumentation Laboratory, Bedford, Massachusetts, United States), a fully automated chemiluminescent immunoassay with an analytical time of 33 minutes. A method comparison study was performed on 176 samples from 49 healthy donors and 127 TMA patients (109 TTP, 7 atypical hemolytic uremic syndrome, 11 other TMAs), comparing this new assay with an in-house FRETS-VWF73 assay and a commercial enzyme-linked immunosorbent assay (ELISA) (TECHNOZYM ADAMTS-13 Activity, Technoclone GmbH, Vienna, Austria). Agreement between methods was assessed with focus on ADAMTS13 activity less than $10 \%$, the medical decision level relevant for TTP diagnosis. The HemosIL AcuStar ADAMTS13 Activity showed good correlation with both the FRETSVWF73 $(r=0.96)$ and ELISA $(r=0.96)$ methods. Slope of the Passing-Bablok regression was 1.05 for FRETS-VWF73 and 1.02 for ELISA, and absolute bias at the medical decision level was +0.1 and $+0.3 \%$, respectively. The study also revealed high agreement with FRETS-VWF73 (kappa 0.97) and ELISA (kappa 0.98) methods in classifying TTP patients received

March 12, 2019

accepted after revision July 13, 2019
DOI https://doi.org/ $10.1055 / \mathrm{s}-0039-1696718$ ISSN 0340-6245. (c) 2019 Georg Thieme Verlag KG Stuttgart · New York
License terms

() (1) $\Theta \circledast$ 
with a severe deficiency of ADAMTS13 activity. Because of its short turnaround time and full automation, the HemosIL AcuStar ADAMTS13 activity assay might become the assay of choice to rapidly test ADAMTS13 activity in plasma and thus establish the diagnosis of acute TTP in emergency settings.

\section{Introduction}

Thrombotic thrombocytopenic purpura (TTP) is a rare and lifethreatening thrombotic microangiopathy (TMA) characterized by mechanical hemolytic anemia, consumption thrombocytopenia, and highly variable symptoms related to end-organ ischemic damage caused by microvascular thrombosis. The hallmark of TTP is a severe deficiency of ADAMTS13 (a disintegrin and metalloprotease with thrombospondin motifs, 13) $\operatorname{activity}$ ( $<10 \%$ of normal). Hence, its measurement is pivotal to confirm a TTP diagnosis and for optimal patient management during acute and remission phases. ${ }^{1}$ However, the utility of ADAMTS13 activity results is hampered by long turnaround times. ${ }^{2}$ Thus, in most instances, the initial diagnosis of TTP remains based on clinical criteria and therapeutic plasma exchange (PEX) is often initiated as soon as TTP is suspected, even before the measurement of ADAMTS13 activity, due to the high mortality if the disease is left untreated.

The HemosIL AcuStar ADAMTS13 Activity assay (Instrumentation Laboratory, Bedford, Massachusetts, United States), is a fully automated chemiluminescent immunoassay (CLIA) with an analytical time of 33 minutes for the quantitative measurement of ADAMTS13 activity in human-citrated plasma on the ACL AcuStar analyzer. ${ }^{3}$ The aim of this study was to evaluate the performance of this assay in comparison to an inhouse FRETS-VWF73 assay and a commercial enzyme-linked immunosorbent assay (ELISA) (TECHNOZYM ADAMTS-13 Activity, Technoclone GmbH, Vienna, Austria).

\section{Materials and Methods}

\section{Study Samples}

The samples included in this report were analyzed in two separate evaluation studies. The first study included the analysis of 76 samples (21 healthy subjects, 46 TTP, 5 atypical hemolytic uremic syndrome [aHUS], and 4 other TMAs) and was performed during January to February 2017. The second study was performed during June to July 2018 and included 112 samples ( 37 healthy subjects, 64 TTP, 4 aHUS, and 7 nonTTP TMA) among which 12 had been tested also during the first study. For the purpose of this report, the results obtained in the first study for these duplicate samples were excluded, thus leaving a total of 176 plasma samples including 49 samples from healthy subjects (blood donors with no records of coagulation or hemorrhagic problems), 100 with acquired TTP (12 in the acute phase and 88 in remission), 9 with congenital TTP, 7 with aHUS, and 11 from patients with other TMAs. Samples were collected between 2006 and 2018 at the Angelo Bianchi Bonomi Hemophilia and Thrombosis Center of Fondazione IRCCS Ca' Granda Ospedale Maggiore Policlinico in Milan and the study was performed in accordance with the principles of the Declaration of Helsinki and with the 21 CFR 50 Protection of Human Patients and 21 CFR 56 Institutional Review Boards. Samples were collected in 3.2\% buffered citrate solution (1:9 anticoagulant:whole blood ratio) and centrifuged at $3,200 \times \mathrm{g}$ for 20 minutes at $4^{\circ} \mathrm{C}$ within 2 hours of collection. Plasma was separated, aliquoted, and stored at $-80^{\circ} \mathrm{C}$ until testing. Plasma aliquots were then thawed for 5 minutes at $37^{\circ} \mathrm{C}$ prior to testing.

\section{Laboratory Tests for ADAMTS13 Activity}

The HemosIL AcuStar ADAMTS13 Activity assay is a fully automated, two-step CLIA. Reagent cartridges include a vial with magnetic particles coated with the VWF73 peptide which contains the ADAMTS13 cleavage site. These particles are incubated with the plasma sample and, after a magnetic separation and a wash step, the chemiluminescent detection of the fragments generated by ADAMTS13 is based upon an isoluminol-labeled monoclonal antibody that specifically reacts with the cleaved peptide. ${ }^{4}$ After this second incubation, a magnetic separation and a wash step, reagents that trigger the chemiluminescent reaction are added. The emitted light is proportional to the ADAMTS13 activity in the sample and is measured as relative light units by the ACL AcuStar analyzer. ${ }^{3}$

ADAMTS13 activity was measured simultaneously in plasma samples with the HemosIL AcuStar ADAMTS13 Activity assay, the commercial ELISA TECHNOZYM ADAMTS-13 Activity, and an in-house FRETS-VWF73 assay. ${ }^{5,6}$ All three assays were standardized against the World Health Organization first International Standard ADAMTS13 Plasma (12/252). - Table 1 shows the main features of these assays.

\section{Statistical Analysis}

A method comparison was performed involving the three aforementioned assays. For calculations, results in $\mathrm{IU} / \mathrm{mL}$ obtained with the TECHNOZYM ADAMTS-13 Activity ELISA were transformed into \% of normality ( $1 \mathrm{IU} / \mathrm{mL}=100 \%$ ). Correlation was assessed using the Pearson's method. For quantitative comparisons, values below the lower limit of the assay range (see - Table 1) were assigned a value equal to $50 \%$ of this limit. Analysis was performed using the PassingBablok regression to calculate the slope, intercept, and their correspondent confidence intervals (CIs). The Bland-Altman analysis was used to investigate systematic differences between methods. The agreement between the HemosIL AcuStar ADAMTS13 Activity assay, the FRETS-VWF73 assay, or the TECHNOZYM ADAMTS-13 Activity ELISA to classify results as lower than $10 \%$, that is, the medical decision level relevant for a TTP diagnosis, was assessed using kappa statistics. Analyzeit v4.60 (Analyze-it Software, Ltd, Leeds, United Kingdom) was used for calculations. 
Table 1 Main features of the ADAMTS13 activity assays

\begin{tabular}{|l|c|c|c|}
\hline & FRETS-VWF73 & $\begin{array}{c}\text { TECHNOZYM ADAMTS-13 } \\
\text { Activity ELISA }^{\text {a }}\end{array}$ & $\begin{array}{c}\text { HemosIL AcuStar } \\
\text { ADAMTS13 Activity }\end{array}$ \\
\hline Substrate & FRETS-VWF73 & GST-VWF73 & GST-VWF73-His \\
\hline Detection method & $\begin{array}{c}\text { Fluorescent: } \\
\text { direct detection of VWF } \\
\text { cleavage products }\end{array}$ & $\begin{array}{c}\text { Chromogenic: } \\
\text { immune-detection of VWF } \\
\text { cleavage products by specific mAb }\end{array}$ & $\begin{array}{c}\text { Chemiluminescent: } \\
\text { immune-detection of VWF } \\
\text { cleavage products by specific mAb }\end{array}$ \\
\hline Detection limit & $3.3 \%$ & $0.002 \mathrm{IU} / \mathrm{mL}(0.2 \%)$ & $0.2 \%$ \\
\hline Assay range & $3.3-105 \%$ & $0.003-1.05 \mathrm{IU} / \mathrm{mL}(0.3-105 \%)$ & $0.2-150 \%$ \\
\hline Intra-assay precision (CV) & $6.0 \%$ & $\leq 5.4 \%$ & $\leq 4.4 \%$ \\
\hline Interassay precision (CV) & $9.5 \%$ & $\leq 8.0 \%$ & $\leq 5.1 \%$ \\
\hline Normal range & $45-147 \%$ & $0.40-1.30 \mathrm{IU} / \mathrm{mL}(40-130 \%)$ & $67-129 \%$ \\
\hline Assay lengthc & 1 hour & $\sim 3.5 \mathrm{hours}$ & 33 minutes \\
\hline
\end{tabular}

Abbreviations: CV, coefficient of variation; mAb, monoclonal antibody; VWF, von Willebrand factor.

a Package insert information.

${ }^{\mathrm{b}}$ The quantitation limit of the HemosIL AcuStar ADAMTS13 Activity assay was revised to a final value of $0.2 \%$ after validation studies completion.

${ }^{c}$ Considering only the time needed to run the assay, excluding sample and calibrator dilution and data analysis.

\section{Results}

\section{Comparison of HemosIL AcuStar ADAMTS13 Activity} versus In-House FRETS-VWF73

A good correlation was obtained for the 176 samples $(r=0.956$, 95\% CI: 0.942-0.967, $p<0.0001$ ) ( Fig. 1 A, upper panel). Slope and intercept values of the Passing-Bablok regression were 1.05 (95\% CI: $0.99-1.14$ ) and -0.41 (95\% CI: -1.36 to 2.05 ), respectively. Absolute bias at $10 \%$ of ADAMTS13 activity was $+0.1 \%$, with a $95 \% \mathrm{Cl}$ of -0.6 to $2.4 \%$ (i.e., an expected value of 9.4-12.4\% ADAMTS13 activity). Bland-Altman analysis showed a mean bias of $-15.9 \%$ (95\% CI: -26.2 to $-5.6 \%$ ), with
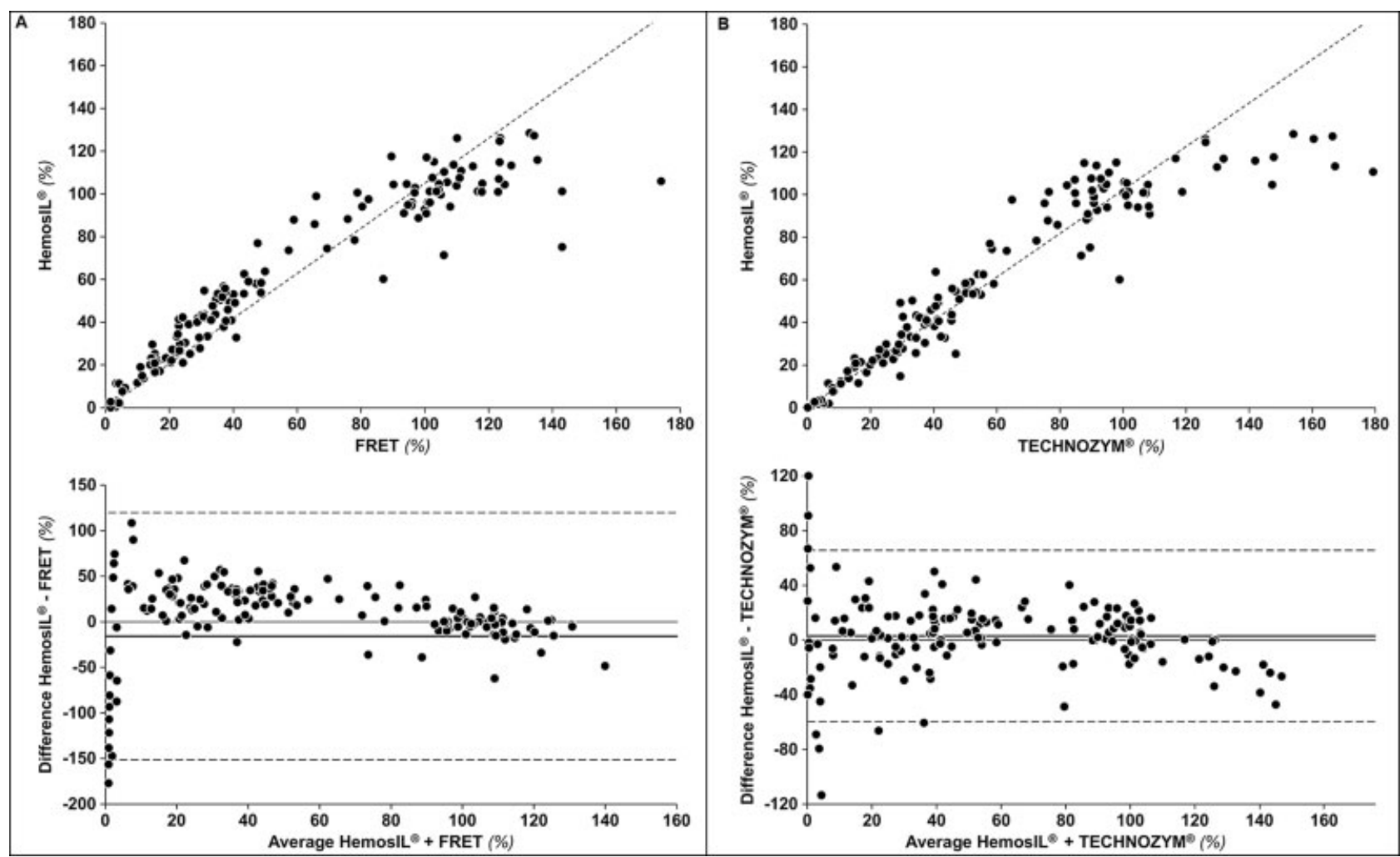

Fig. 1 Results of the comparison between assays. (A) Comparison between HemosIL AcuStar ADAMTS13 Activity (HemosIL) and in-house FRETS-VWF73 (FRET). Upper panel, the Passing-Bablok regression plot. Dashed line: fitted regression line $y=-0.41+1.05 \times$. Lower panel, the Bland-Altman plot. Solid line: mean bias = $-15.9 \%$; dashed lines: $95 \%$ limits of agreement -152 to 120\%. (B) Comparison between HemosIL AcuStar ADAMTS13 Activity (HemosIL) and TECHNOZYM ADAMTS-13 Activity ELISA (TECHNOZYM). Upper panel, the Passing-Bablok regression plot. Dashed line: fitted regression line $y=0.05+1.02 \times$. Lower panel, the Bland-Altman plot. Solid line: mean bias $=2.9 \%$; dashed lines: $95 \%$ limits of agreement -60 to $66 \%$. 
Table 2 Agreement between HemosIL AcuStar ADAMTS13 Activity with FRETS-VWF73 and TECHNOZYM ADAMTS-13 Activity ELISA

\begin{tabular}{|l|c|c|c|c|}
\hline & \multicolumn{2}{|c|}{ FRETS-VWF73 } & \multicolumn{2}{c|}{ TECHNOZYM ADAMTS-13 Activity ELISA } \\
\hline $\begin{array}{l}\text { HemosIL AcuStar } \\
\text { ADAMTS13 Activity }\end{array}$ & $\begin{array}{c}<10 \% \\
(n=46)\end{array}$ & $\begin{array}{c}\geq 10 \% \\
(n=130)\end{array}$ & $\begin{array}{c}<10 \% \\
(n=45)\end{array}$ & $\begin{array}{c}\geq 10 \% \\
(n=131)\end{array}$ \\
\hline$<10 \%(n=44)$ & 44 & 0 & 44 & 0 \\
\hline$\geq 10 \%(n=132)$ & 2 & 130 & 1 & 131 \\
\hline Kappa coefficient & \multicolumn{2}{|c|}{0.97} & \multicolumn{2}{c|}{0.98} \\
\hline
\end{tabular}

limits of agreement (LoA) of -152 and $120 \%$ ( - Fig. 1A, lower panel). This result indicates that, on average, the HemosIL assay reports lower activity values than the FRETS-VWF73 assay. However, this was due to samples with ADAMTS13 activity levels below the limit of quantitation of the FRETS-VWF73 assay. Indeed, when the analysis was restricted to the 139 samples with measurable activity levels, the mean bias was 12.3\% (95\% CI: 7.3-17.3\%), reflecting an average overestimation by the HemosIL assay. This was especially observed in the intermediate-low activity range (from the limit of quantitation to 70\%)( - Fig. 1 A, lower panel). Finally, the study revealed high agreement (kappa $=0.97$ ) between the HemosIL AcuStar ADAMTS13 Activity and the FRETS-VWF73 assays in classifying samples with severe deficiency $(<10 \%)$ of ADAMTS13 activity (-Table 2).

\section{Comparison of HemosIL AcuStar ADAMTS13 Activity versus TECHNOZYM ADAMTS-13 Activity}

Correlation was good $(r=0.957,95 \%$ CI: 0.943-0.968, $p<0.0001$ ) between the TECHNOZYM ADAMTS-13 Activity ELISA and the HemosIL AcuStar ADAMTS13 Activity for the 176 samples included in this study (-Fig. 1B, upper panel). Slope and intercept values of the Passing-Bablok regression were 1.02 (95\% CI: $0.99-1.07)$ and 0.05 (95\% CI: -0.06 to 0.25 ), respectively. Absolute bias at $10 \%$ of ADAMTS13 activity was $0.3 \%$, with a $95 \% \mathrm{CI}$ of -0.05 to 0.7 (i.e., an expected value of 10.0-10.7\% ADAMTS13 activity). The Bland-Altman analysis revealed a mean bias of $2.9 \%$ (95\% CI: -1.8 to $7.7 \%$ ), with LoA of -60 and $66 \%$ ( - Fig. 1B, lower panel). A trend for the HemosIL assay to underestimate activity values in the high assay range $(>110 \%)$ was observed. A high degree of agreement (kappa $=0.98$ ) was obtained between the HemosIL AcuStar ADAMTS13 Activity assay and the commercial ELISA in classifying samples with $<10 \%$ ADAMTS13 activity (-Table 2).

\section{Diagnostic Accuracy and Analytical Sensitivity of HemosIL AcuStar ADAMTS13 Activity}

-Fig. 2 shows the distribution of the HemosIL AcuStar ADAMTS13 Activity results according to the corresponding clinical diagnosis for the 176 plasma samples analyzed. Concordantly with FRETS-VWF73 and TECHNOZYM ADAMTS-13 Activity, the HemosIL AcuStar ADAMTS13 Activity assay reported severely reduced levels of ADAMTS13 activity ( $<10 \%)$ for all patients with acquired TTP in the acute phase and for all but one patient with congenital TTP (HemosIL AcuStar ADAMTS13 Activity 11.4\%, TECHNOZYM
ADAMTS-13 Activity 6.6\%, and FRETS-VWF73 3.4\%). Concordance between assays was observed in the detection of ADAMTS13 activity levels in all but one patient with acquired TTP in the remission phase (HemosIL AcuStar ADAMTS13 Activity $11.3 \%$, TECHNOZYM ADAMTS-13 Activity $10.6 \%$, and FRETS-VWF73 4.3\%), in healthy donors and in patients with aHUS or other TMAs.

The detection capability of the HemosIL AcuStar ADAMTS13 Activity assay allowed better resolution of the very low ADAMTS13 activity samples in comparison with the FRETS-VWF73 (-Fig. 3). Forty-four samples included in the study gave ADAMTS13 activity levels below $10 \%$ with the three methods. The HemosIL AcuStar ADAMTS13 Activity allowed measurement of ADAMTS13 activity in 31 of them (70\%) to a minimum value of $0.2 \%$, in comparison with 16 samples (36\%) for the TECHNOZYM ADAMTS-13 Activity to a minimum value of $0.2 \%$ and 7 samples (16\%) for the FRETSVWF73 to a minimum value of $3.3 \%$.

\section{Discussion}

In this study, we evaluated the performance of a new method, the HemosIL AcuStar ADAMTS13 Activity assay, in a large population of patients with TTP, aHUS, other TMAs, and in healthy controls. The new assay showed significant correlation with the in-house FRETS-VWF73 assay and the TECHNOZYM ADAMTS-13 Activity ELISA. At Passing-Bablok

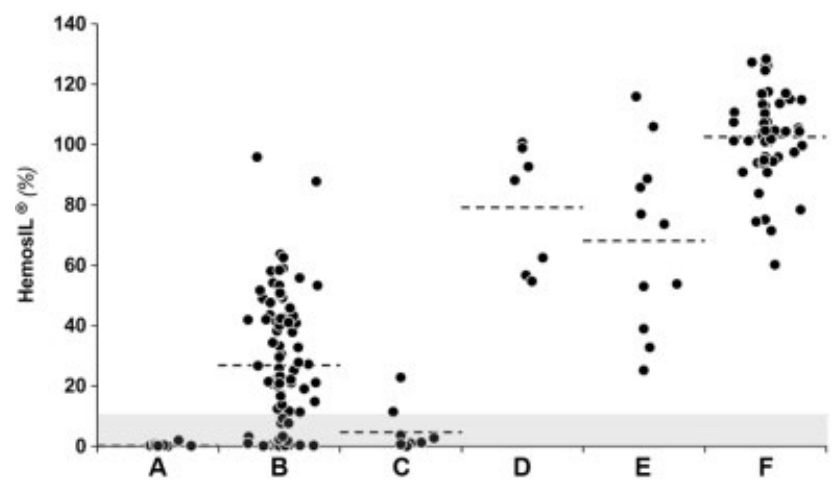

Fig. 2 Distribution of results of HemosIL AcuStar ADAMTS13 Activity according to the diagnosis. Dashed lines show the mean ADAMTS13 activity value obtained for each group of samples. The 10\% ADAMTS13 activity level, used to define the cutoff for severe ADAMTS13 activity deficiency, is also shown (gray area). (A) Acquired thrombotic thrombocytopenic purpura (TTP) in acute phase. (B) Acquired TTP in remission phase. (C) Congenital TTP. (D) atypical hemolytic uremic syndrome (aHUS). (E) Other thrombotic microangiopathies (TMAs). (F) Normal. 


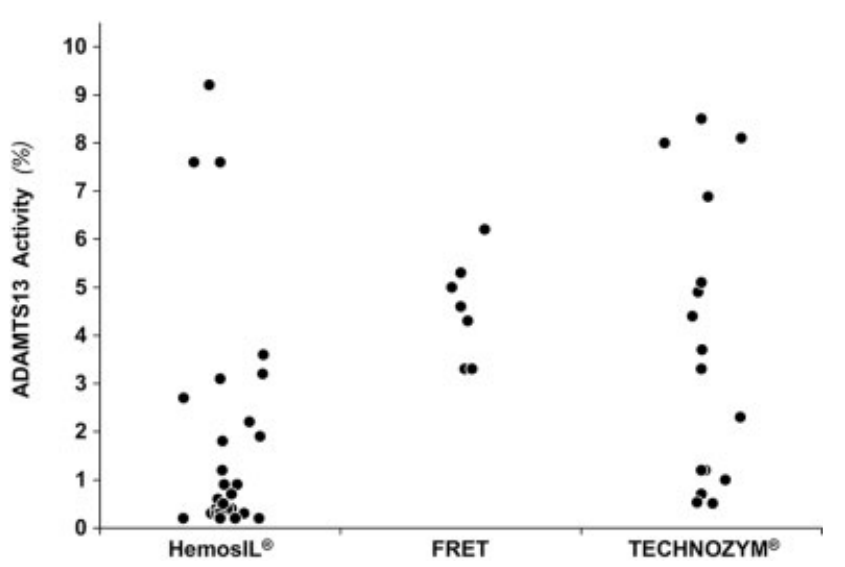

Fig. 3 Number of samples accurately quantified by each assay among the 44 with ADAMTS13 activity levels below 10\%: 31 with the HemosIL AcuStar ADAMTS13 Activity (HemosIL), 7 with the FRETS-VWF73 (FRET), and 16 with the TECHNOZYM ADAMTS-13 Activity ELISA (TECHNOZYM).

regression analysis, no constant differences and good comparability with both methods were observed. Moreover, the HemosIL AcuStar ADAMTS13 Activity assay showed a high agreement with the FRETS-VWF73 and TECHNOZYM assays in detecting ADAMTS13 activity levels below 10\%, which define the internationally accepted cutoff for a diagnosis of severe ADAMTS13 deficiency typical of TTP. ${ }^{1}$

The analytical performance of the HemosIL AcuStar ADAMTS13 Activity assay in comparison with the TECHNOZYM ADAMTS-13 Activity assay has already been evaluated by Favresse et al in a relatively small series of 38 plasma samples from patients with severe $(n=8)$, moderate $(n=2)$, and normal $(n=28)$ ADAMTS13 activity levels. ${ }^{7}$ The authors reported on average lower levels as measured by the HemosIL assay (mean difference of HemosIL and TECHNOZYM assay results: $-4.8 \%$ [95\% CI: -9.83 to $0.27 \%$ ]). This is somewhat in contrast with our findings, which indicate on average higher levels as measured by the HemosIL assay (mean bias 2.9\% [95\% CI: -1.8 to $7.7 \%$ ]). Differences in the reagent lots and in the type and number of samples tested (the full range of ADAMTS13 activity values is more represented in our study) may explain this minor difference. Most importantly, as in our study, Favresse et al found a full agreement between the two assays in detecting severe ADAMTS13 deficiency, with no misclassification and a bias close to zero when considering only ADAMTS13 activity values below $10 \%{ }^{7}$

TTP is a life-threatening condition often presenting with rapid and severe progression, and thereby should be diagnosed and treated in emergency with PEX, as soon as it is suspected. The rather long turnaround times and complex execution of commonly used methods for ADAMTS13 measurement, which include the FRETS-VWF73 and TECHNOZYM ADAMTS-13 Activity assays, often lead clinicians to decision-making in situations of emergency without the support of ADAMTS13 test results. Although the HemosIL assay requires the AcuStar instrumentation, which has a rather limited diffusion in comparison with spectrophotometric devices, the more rapid turnaround time of this assay favors a prompt and accurate diagnosis. More certainty regarding an accurate diagnosis of
TTP using this assay would also, in addition to the use of PEX, allow consideration for the early use of caplacizumab, with the related potential benefits. ${ }^{8}$

Our study has limitations. First, our sample population has been collected between 2006 and 2018, raising the issue of a potential differential effect of prolonged frozen storage on ADAMTS13 activity measurements obtained with the three analyzed assays. However, the vast majority of samples (85\%) were stored for less than 3 years, $52 \%$ for less than 1 year. No difference in the correlation analysis was observed after stratification on the basis of the duration of storage (-Supplementary Table S1, available in the online version). Another limitation of our study is that we did not measure ADAMTS13 activity on fresh plasma samples, as it would be done in emergency settings. Although this does not invalidate the comparisons herein performed, we are unable to establish whether testing fresh or biobanked frozen samples would differentially affect the ADAMTS13 activity results obtained by the three assays. Further studies are required to investigate this issue.

In conclusion, the results reported herewith indicate that full automation and short turnaround time of the new HemosIL AcuStar ADAMTS13 Activity assay provide important characteristics for use in emergency settings, even in hospital laboratories without specialized personnel.

\section{What is known about this topic?}

- Thrombotic thrombocytopenic purpura is a rare, lifethreatening thrombotic microangiopathy characterized by the severe deficiency of ADAMTS13 activity ( $<10 \%)$.

\section{What does this paper add?}

- The performance of the fully automated HemosIL AcuStar ADAMTS13 activity assay with an analytical time of 33 minutes was evaluated.

- The new assay showed excellent clinical performance in detecting severe ADAMTS13 deficiency and good correlation with in-house FRETS-VWF73 assay and TECHNOZYM ADAMTS-13 Activity ELISA.

- Acute thrombotic thrombocytopenic purpura diagnosis in emergency setting can benefit from this assay.

Authors' Contributions

C.V. designed the study, performed experiments, performed quality control, interpreted the results, and wrote the manuscript. M.M. developed the HemosIL AcuStar ADAMTS13 Activity assay, designed the study, performed quality control, interpreted the results, and wrote the manuscript. I.M. designed the study, interpreted the results, and critically reviewed the manuscript. M.B. collected clinical and/or experimental data and performed experiments and critically reviewed the manuscript. L.S. collected clinical and/or experimental data and critically reviewed the manuscript. 
S.F. designed the study, analyzed and interpreted the results, and critically reviewed the manuscript. D.M.-P. designed the study, analyzed and interpreted the results, and critically reviewed the manuscript. D.G. designed the study, interpreted the results, and critically reviewed the manuscript. J.S.-D. interpreted the results and critically reviewed the manuscript. S.B. developed the HemosIL AcuStar ADAMTS13 Activity assay, collected experimental data, and critically reviewed the manuscript. S.M.T. enrolled patients, and critically reviewed the manuscript. L.F. enrolled patients and critically reviewed the manuscript. E.R. enrolled patients and critically reviewed the manuscript. F.P. designed the study, enrolled patients, interpreted the results, and critically reviewed the manuscript. All authors approved the final version of the manuscript.

\section{Conflict of Interest}

F.P. has received honoraria for participating as a speaker at satellite symposia and educational meetings organized by Ablynx, Grifols, Novo Nordisk, Roche, Shire, and Sobi. She has received consulting fees from Kedrion and she is recipient of a research grant funding from Biokit paid to Fondazione Luigi Villa. She is member of the scientific advisory board of Ablynx. I.M. received personal fees by Sanofi for participating in a Sanofi Advisory Board meeting. C.V., M.B., and L.S. are affiliated with Fondazione Luigi Villa, that received financial support from Biokit for the present work. M.M., S.F., D.M.-P., D.G., J.S.-D., and S.B. are employed at Biokit that is the developer of the assay in evaluation. The other authors do not have any conflict of interests to disclose.

\section{Acknowledgments}

The authors gratefully acknowledge Prof. P. M. Mannucci for his precious help and fruitful discussion, and Dr. L. F. Ghilardini for helping with figures.

\section{References}

1 Scully M, Cataland S, Coppo P, et al; International Working Group for Thrombotic Thrombocytopenic Purpura. Consensus on the standardization of terminology in thrombotic thrombocytopenic purpura and related thrombotic microangiopathies. JThromb Haemost 2017;15(02):312-322

2 Connell NT, Cheves T, Sweeney JD. Effect of ADAMTS13 activity turnaround time on plasma utilization for suspected thrombotic thrombocytopenic purpura. Transfusion 2016;56(02):354-359

3 Mirabet M, Blanch S, Puig J, Serra J. Highly sensitive fully automated chemiluminescent immunoassay for rapid quantification of ADAMTS13 activity. Res Pract Thromb Haemost 2017;1(Suppl 1):1297

4 Kato S, Matsumoto M, Matsuyama T, Isonishi A, Hiura H, Fujimura Y. Novel monoclonal antibody-based enzyme immunoassay for determining plasma levels of ADAMTS13 activity. Transfusion 2006;46(08):1444-1452

5 Kokame K, Nobe Y, Kokubo Y, Okayama A, Miyata T. FRETS-VWF73, a first fluorogenic substrate for ADAMTS13 assay. Br J Haematol 2005; 129(01):93-100

6 Mancini I, Valsecchi C, Lotta LA, et al. FRETS-VWF73 rather than CBA assay reflects ADAMTS13 proteolytic activity in acquired thrombotic thrombocytopenic purpura patients. Thromb Haemost 2014;112(02):297-303

7 Favresse J, Lardinois B, Chatelain B, Jacqmin H, Mullier F. Evaluation of the fully automated HemosIL Acustar ADAMTS13 Activity assay. Thromb Haemost 2018;118(05):942-944

8 Scully M, Cataland SR, Peyvandi F, et al; HERCULES Investigators. Caplacizumab treatment for acquired thrombotic thrombocytopenic purpura. N Engl J Med 2019;380(04):335-346 